Voix et Images

voixetimages

\title{
Suzanne Lamy, pour une morale de la critique
}

\section{Lori Saint-Martin}

Volume 13, numéro 1 (37), automne 1987

Suzanne Lamy

URI : https://id.erudit.org/iderudit/200681ar

DOI : https://doi.org/10.7202/200681ar

Aller au sommaire du numéro

\section{Éditeur(s)}

Université du Québec à Montréal

\section{ISSN}

0318-9201 (imprimé)

1705-933X (numérique)

Découvrir la revue

\section{Citer cet article}

Saint-Martin, L. (1987). Suzanne Lamy, pour une morale de la critique. Voix et Images, 13(1), 29-40. https://doi.org/10.7202/200681ar d'utilisation que vous pouvez consulter en ligne.

https://apropos.erudit.org/fr/usagers/politique-dutilisation/ 


\title{
Suzanne Lamy, pour une morale de la critique
}

\author{
par Lori Saint-Martin, Université Laval
}

Tant il n'est d'esthétique qui ne renvoie à une éthique...

Suzanne Lamy, «D'un autre lieu commun»

C'est en femme que je veux être lue et entendue. Suzanne Lamy, Quand je lis je m'invente

Suzanne Lamy s'est surtout fait remarquer par ses livres et ses conférences sur l'écriture des femmes, au point de devenir l'experte attitrée en la matière. Mais ce volet de son oeuvre de critique, aussi essentiel soit-il, ne doit pas occulter sa vision globale de l'écriture et de la critique, vision esthétique et morale d'une rare perspicacité.

Ses amours littéraires auront été nombreuses. D'abord, le surréalisme, sa thèse de doctorat sur Arcane 17, d'André Breton, l'anthologie de textes québécois marqués par le surréalisme qu'elle préparait à la fin de sa vie. Marguerite Duras, Luce Irigaray, la modernité en littérature québécoise, les revues culturelles, le passage de la voix dans les écrits, le retour du référent, les lieux de pouvoir, autant d'interrogations qui l'auront retenue à un moment ou l'autre de sa vie. Comme directrice et critique, à Spirale, elle prend position sur l'actualité littéraire, par passion ou par impatience, d'une voix à la fois personnelle et forte de solides acquis théoriques.

Elle refuse la dichotomie facile qu'on établit entre rationnel et senti, autrement dit, entre masculin et féminin:

ce n'est pas vrai que d'un côté il y a la raison, la logique, l'intellect, la raideur, le sec... et de l'autre l'intuition, l'irrationnel, la pensée magique, la sensibilité, la douceur, l'humide...1

Elle revendique plutôt le libre passage entre les deux pôles. On la connaît en effet aussi bien pour des textes «humides» sur les écrits qui la ravissent Breton, Duras, des écrits de femmes - que pour des textes «secs», durs, qui démolissent dans les règles les Fous de Bassan, la Constellation du Cygne et quelques autres.

1 Suzanne Lamy, Quand je lis je m'invente, Montréal, l'Hexagone,1984, p. 26. 
Afin de dire les attaches de Suzanne Lamy, ses préoccupations, la forme de sa trajectoire critique, j'adopterai une démarche voisine de la sienne. Comme elle, $j^{\prime}$ irai a mon allure quelque peu serpentine... ${ }^{2}$, en relevant, dans le fouillis des textes, ce qui me semble être l'essentiel. Amoureuse, ma critique le sera à son tour.

\section{Une subjectivité revendiquée}

D'où Suzanne Lamy nous parle-t-elle, elle qui, à la suite de Bourdieu, fait remarquer à de nombreuses reprises que le lieu d'où l'on parle compte souvent davantage dans nos sociétés que la parole elle-même 3 ?

Avant tout, elle annonce, proclame sa différence: C'est en femme que je veux être lue et entendue 4 . Je suis entraînée; je suis écartelée $e^{5:}$ la présence du «je» dans ses écrits, ce «e» final nullement muet dans un contexte où il n'en finit pas de surprendre, fait sa marque. Loin de croire naïvement qu'on peut éviter les partis pris, elle déclare d'entrée de jeu les siens, dont le mépris du discours universitaire faussement universel: Toute lecture n'impliquerait-elle pas des a priori, avoués ou non, et les hommes seraient-ils des anges 6 ?

Des anges, elle n'en veut d'ailleurs pas, mais des voix, des êtres de chair: ...l'impression que, dans le texte, circule un esprit, passe une présence...7. Elle réclame ainsi, non pas une subjectivité bon marché, mais l'engagement total du critique dans ce qu'il produit. Le sérieux de la recherche et de la méthode sont indispensables, mais aussi, la saveur de l'écriture.

Ma lecture, je la voudrais parole qui ouvre les cages de l'écrivain-écrivant et du lecteur-critique ${ }^{8}$, écrit Suzanne Lamy. Ainsi s'accorde-t-elle la liberté d'écrire avec le texte plutôt que sur le texte ${ }^{9}$, se faisant pétillante pour errer dans le labyrinthe de la Vie en prose, minutieuse et patiente pour déchiffrer la petite phrase de France Théoret: Je suis un noeud ${ }^{10}$. La liberté de jouer avec les mots, elle la prend aussi.

2 Ibid., p. 30.

3 Ibid., p. 101.

4 Ibid., p. 26.

5 Suzanne Lamy, «Au jeu du désir et de la mort», dans Marcelle Brisson, Eros au pluriel, Montréal, HMH, 1984, p. 64-66.

6 Quand je lis je m'invente, p. 30.

7 Suzanne Lamy, «Au-delà de l'actualité», dans Voix \& images, 34, vol. XII, no 1 , automne 1986 , p. 161.

8 Quand je lis je m'invente, p. 27.

9 Suzanne Lamy et Irène Pagès, textes réunis et présentés par, Féminité, subversion, écriture, Montréal, Remue-ménage, 1983, p. 5.

10 Suzanne Lamy, «Subversion en rose», «Des résonnances de la petite phrase: Je suis un noeud, de France Théoret», dans Féminité..., p. 107-118, 139-149. 
Ce qui ne la rend ni narcissique, ni complaisante. Son écriture porte la marque de celle qui l'a produite, sans bavures ni indiscrétion. Personne de plus modeste qu'elle, de plus ouverte à l'admiration. Ses textes sont personnels, mais non entachés d'égotisme. Dans Spirale, par exemple, elle donne des articles journalistiques, solidement construits et pleins de renseignements, mais elle en a signé peu où il ne passe quelque chose d'autre, de plus, qui fait sa griffe: une métaphore qu'elle s'amuse à filer (celle de la nourriture s'est révélée particulièrement féconde), des mots rares ou savoureux, une construction peu usitée. Ou bien elle fait un retour éclairant à l'étymologie, à propos de la litanie ou du bavardage, dans d'elles, ou une rêverie sur le mot «transmission», pour la revue Critère.

\section{Un acte d'amour}

Écrire est un acte d'amour. De soi. Des autres ${ }^{11}$, écrit Suzanne Lamy dans un texte de fiction paru dans la Nouvelle Barre du jour. Ces amours-là, elle en a parlé de manière exemplaire.

Séduisent plus que tout autre qualité chez elle la passion de l'écriture, la capacité d'envoûtement: Livres léchés, mâchés, ma nourriture. Livres que je fais miens par l'écho, la gratitude12.

Quels livres aime-t-elle au juste? Ceux dont la lecture brûle et transforme, ceux qui ont le pouvoir de féconder et aussi de faire perdre l'équilibre13. Les textes limites, comme ceux de ses grandes amours, Breton et Duras, qui n'ont de poids que si le lecteur veut bien, lui aussi, raser les précipices, suivre le désir qui ne connaût plus de digues, d'où l'on ne revient que les os broyés 14 .

Sans cesse, Suzanne Lamy en revient à son amour des textes ou il y a réellement écriture 15 , au sens de Barthes. Elle refuse l'informe, le brouillon, les fragments donnés en vrac, au hasard des béances et des états d'âme...16. Le témoignage la sollicite, mais seulement lorsqu'il y a en même temps travail sur la forme. Elle critique également certains écrits de femmes, confus, décousus, faits à la va-comme-je-te-pousse, publiés sous le couvert d'une spontanéité prétendument «naturelle», comme si l'écrit pouvait couler de source ${ }^{17}$. Tout ce qui est brut, sans écriture, la laisse aussi sans désir.

11 «Messe en si», dans la Nouvelle Barre du jour, no 106, octobre 1981, p. 81.

12 Quand je lis je m'invente, p. 9.

13 «Au jeu du désir...», p. 85.

14 Quand je lis je m'invente, p. 70.

15 Suzanne Lamy, "Grandeurs et misères de la critique au féminin», présenté à l'Université Concordiax-- Institut Simone de Beauvoir, février 1984, p. 1 du manuscrit.

16 Suzanne Lamy, «Quand le silence est d'or», dans Spirale, no 23, mars 1982, p. 6.

17 Suzanne Lamy, «Excellent, MAIS...», dans Spirale, no 30, décembre 1982, p. 7 . 
La très haute estime dans laquelle Suzanne Lamy tient l'écriture la rend exigeante. Ecrire, c'est tout de même autre chose que tenir un journal de collège ${ }^{18}$, rappelle-t-elle avec sévérité; mieux, il n'y a aucune obligation à écrire19.

Ainsi se consacre-t-elle aux textes qui en valent réellement la peine: Ambiguë et immense, l'écriture garde son pouvoir dans la mesure où il y a travail sur le langage, d̀ l'intérieur d'une recherche qui est en prise sur l'histoire 20. Les écrits des femmes et les textes modernes québécois retiennent son attention en raison du souci d'écriture, du goût du mouvant, du risque, et du très grand désir d'exploration qui les animent 21 .

La densite, l'enchevêtrement des textes modernes la ravissent. Comme elle le rappelle, certaine difficulté a partie liée avec ce qui a nom d'écriture même si la résistance du texte ne donne pas de garantie sur sa qualité22.

Revient souvent, pour caractériser les textes aimés, la double métaphore de la voix et de la tresse. De la dramatique de Monique La Rue, l'Enregistrement, Suzanne Lamy retient ce que la voix peut produire de désir, de jouissance, dans l'entrelacement de la vie privée et de la trame politique, ce tissage serré, inextricable, impossible à défaire, qui nous concerne tous et toutes 23 .

La présence de la voix lui semble marquer les meilleurs écrits des femmes: Peut-être appartient-il davantage à certaines femmes aujourd'hui de ne pas couper leur voix de leur corps, de ne pas cacher combien leur corps entre en résonance à certains timbres ${ }^{24}$.

Comment répondre au tissage du texte, sinon par un autre texte aussi finement tramé? Pour sa thèse, Suzanne Lamy avait adopté une approche empirique et intuitive qui rende compte du tissu du texte dont les fils sont si sûrement et en apparence si négligemment tressés 25 . Souvent, elle procède par tissage, par entrelacement de deux auteurs ou deux textes aimés: Duras et Breton, ou l'Homme assis dans le couloir et le Journal d'une femme soumise. À cette

18 Suzanne Lamy, «Une pastille à odeur d'encens», dans Spirale, no 19, mai 1981 , p. 4.

19 «Au-delà de l'actualité», p. 161.

20 Quand je lis je m'invente, p. 20.

21 Ibid., p. 75.

22 IBid., p. 23.

23 Suzanne Lamy, "Au contact d'une voix», présenté au 3e colloque du Southem Council on Francophone Studies, Albuquerque, mars 1985, p. 11 et 9 du manuscrit.

24 «Au jeu du désir...», p. 83.

25 Suzanne Lamy, André Breton, Hermétísme et poésie dans Arcane 17, Montréal, PUM, 1977, p. 22. 
mise en relation, la raison ne trouvera pas son compte 26 : l'essentiel se passe ailleurs. Suzanne Lamy se préoccupe alors de faire parler les textes d'une manière nouvelle, fruit d'une confrontation inattendue:

Lier ces deux textes en un troisieme. Passer de l'une à l'autre de leurs lignes. Les entrelacer. [...] Il faudra trouver des fils, repérer les anfractuosités de ces textes...27.

Pour lire les écrits des femmes québécoises, elle procède de la même manière: Je rassemble et confronte les traces 28 .

\section{Le parcours d'une amoureuse 29}

La résonance, la voix qui sollicite une réponse, voilà ce sur quoi se fonde une critique amoureuse. Il faut accepter d'être subjugué-e par certains textes, se laisser entamer par la lecture jusque dans ses fibres intimes ${ }^{30}$, mais sans perdre sa lucidité. L'éloge facile et la paraphrase ne valent pas plus cher que la critique marquée de raideur et de froideur ${ }^{31}$. Les dangers sont réels, la tentation aussi. Suzanne Lamy a bien mesuré les deux:

Sans méconnaître les risques d'égarement, d'absorption par la relation étroite, pourquoi ne pas choisir le rapport amoureux, celui qui ne veut rien laisser perdre et qui, sans forcément obscurcir la vue, affine l'oreille aux nuances de l'humour comme de la tendresse 32 .

On pense ici, un instant, aux théories de Luce Irigaray (que Suzanne Lamy admirait beaucoup) sur la prédominance de l'ouïe chez les femmes, moins voyeuses que les hommes 33 .

Mais travailler seulement «avec son coeur» ne donne rien qui vaille: la recherche, la méthode sont essentielles. Dans un compte rendu dont le titre, «Du hobby à la publication», en dit long, Suzanne Lamy remarque, ironique: Un coeur qui a de drôles de ratés: un bon stimulateur n'aurait pas été superflu 34 .

Encore une fois, la critique présuppose un engagement total. De neutralité, il n'y en aura pas. Je vous invile seulement au parcours d'une amoureuse ${ }^{35}$. Peu

26 «Au jeu du désir...», p. 64.

27 «Au jeu du désir...», p. 63.

28 Quand je lis je m'invente, p. 9.

29 Ibid., p. 62.

30 Ibid., p. 23.

31 Suzanne Lamy, «Duras maintenant», dans S. Lamy et André Roy, Marguerite Duras à Montréal, Montréal, Spirale, 1981, p. 171.

32 Quand je lis je m'invente, p. 10.

33 Luce Irigaray, Ce sexe qui n'en est pas un, Paris, Minuit, 1977, p. 25.

34 Suzanne Lamy, «Du hobby à la publication...» dans Spirale, no 58, février 1986 , p. 14.

35 Quand je lis je m'invente, p. 62. 
de personnes sont à la hauteur de ce travail exigeant, tant la critique amoureuse est coincée entre le double écueil de la distance et de la complaisance. N'est pas amoureux, ne le raconte pas, qui veut.

\section{Splendeurs et misères de la théorie}

Passionnée de théorie littéraire, Suzanne Lamy se réjouit des lieux où l'émotion est intellectuelle 36 . Sa «méthode» critique varie selon les textes qu'elle aborde, et elle s'impose à chaque fois de nommer ses appuis théoriques. Elle conserve toutefois une certaine distance à l'égard de la théorie, qui a toujours partie liée avec l'idéologie et avec l'institution 37 .

C'est surtout comme lectrice de textes de femmes qu'elle interroge la theorie en tant que pouvoir. Pour rendre compte de cette production, à cause de sa complexité, il faudra emprunter à plusieurs sources, sinon à toutes 38 .

Quels sont donc ses appuis?

...le marxisme pour l'analyse dialectique des aliénations; l'existentialisme [...] pour nous avoir montré qu'il appartient à chacun de se faire, à partir de ce que l'on a fait de nous; le surréalisme, pour l'esprit de révolte et la place donnée d̀ l'amour...39.

Ensuite, la critique sociologique, Bourdieu, Dubois et les autres, pour défaire les idéologies et les modèles, pour déceler les contradictions... ${ }^{40}$.

La psychanalyse fait comprendre des choses, aussi utiles pour la lecture que pour les comportements, sur le déplacement, la condensation, le narcissisme, la dénégation, le masochisme... ${ }^{41}$. Sur la critique transférentielle qu'inspirent Duras ${ }^{42}$, le Journal d'une femme soumise de Mara, ou les écrits des femmes, la psychanalyse apporte de précieux éclaircissements. Mais il convient de se méfier de son conservatisme: elle est un trop bon renfort pour la famille ${ }^{43}$.

La principale référence théorique, toutefois, c'est le structuralisme, et surtout, Roland Barthes, le deuxième, celui du Plaisir du texte et de la Leçon contre le premier 44 . De Barthes, Suzanne Lamy a retenu la définition même de

36 Suzanne Lamy, «Quand l'émotion est intellectuelle», dans Splrale, no 40, février 1984, p. 7.

37 «Grandeurs et misères...», p. 2 du manuscrit.

38 Quand je lis je m'invente, p. 10.

39 Suzanne Lamy, d'elles, Montréal, l'Hexagone, 1979, p. 58.

40 Quand je lis je m'invente, p. 25.

41 Ibid., p. 25-26.

42 Suzanne Lamy, «Marguerite Duras et la critique transférentielle», présenté au Centre culturel canadien, février 1982; voir aussi «Au jeu du désir...», p. 8389.

43 Quand je lis je m'invente, p. 26.

44 «Au jeu du désir...», p. 76. 
l'écriture, la parenté du texte critique et du texte de création, l'importance du plaisir dans l'acte de lecture, la démarche riche d'errances et d'explorations.

Du structuralisme, elle admire la rigueur et la méthode. Mais sur le plan idéologique, cette approche présente une grande défaillance: parce qu'elle fait abstraction de l'Histoire, elle ne peut éclairer les textes féminins, marqués par l'articulation du passé et du présent, du privé et du politique ${ }^{45}$.

Dans les écrits des dernières années, on voit Suzanne Lamy rejeter l'un des principes fondateurs du structuralisme, à savoir le refus du référent. Elle ne croit pas au texte innocent, coupé de toute réalité, donc inattaquable. Elle rappelle que les fondateurs du structuralisme redécouvraient Bataille, Sade et les autres au moment d'élaborer leurs théories: faudrait-il alors s'étonner qu'il y ait quelques rapports de l'ordre des discours mystifiants entre l'oppression des femmes REELLE — 46 et la théorie structuraliste?

D'où l'importance de s'interroger sur les médiations sournoisement tramées qui peuvent exister entre signifiant, référent et réel ${ }^{47}$. Critique politique, donc, critique engagée.

Des travaux récents réalisés dans le domaine de la production et de la réception, notamment autour de la revue Texte, permettent à Suzanne Lamy d'étayer sa conviction profonde qu'on doit tenir compte du référent. Les noms de Linda Hutcheon, de Catherine Kerbrat-Orecchioni, d'Édouard Morot-Sir reviennent souvent. Lui sera également utile la pragmatique, avec Austin et Searle, qui mettent en lumière la situation des interlocuteurs, leurs rapports entre $e u x 48$, mais aussi avec Shoshana Felman, admirée entre autres parce qu'elle ose se dire rséduite» par Austin 49 .

\section{Texte et idéologie}

Pour Suzanne Lamy, esthétique et éthique vont de pair:

Et comme chaque fois qu'il y a démarche esthétique d'une densité certaine, il y a, concomitante, une recherche qui engage la totalité de l'être 50 .

Impossible, donc, de couper le texte de son contexte social et politique: L'approche esthétique a tout a gagner de la connaissance du contexte, des

\footnotetext{
45 Quand je lis je m'invente, p. 43.

46 «Au jeu du désir...», p. 69.

47 «Au jeu du désir...», p. 69.

48 «Grandeurs et misères...», p. 7.

49 «Au jeu du désir...», p. 76.

50 Suzanne Lamy, «D'un autre lieu commun», présenté à l'Université Concordia, 1984, p. 6 du manuscrit.
} 
médiations avec le faire, le toucher, avec l'analyse de l'attente ou du refus du public 51 .

Parallèlement, elle s'insurge contre tous les abus de pouvoir que permet le fait d'écrire. Elle s'indigne de voir que beaucoup de romans dits «féminins» de forme traditionnelle profitent seulement de la récupération de la vague féministe par les éditeurs 52 . C'est le cas, par exemple, d'Erica Jong avec Fanny; dans le domaine critique, une Béatrice Didier «utilise» aussi le féminisme ${ }^{53}$.

La place réduite qu'occupent les femmes dans l'institution littéraire retient aussi l'attention de Suzanne Lamy. Elle pose le problème de l'intégration des écritures au féminin à l'histoire des idées de ce siècle ${ }^{54}$, déplore que ces écritures soient étudiées en parasite avec ce qui constitue l'épine dorsale de l'enseignement et de la recherche 55 .

Mais elle s'inquiète aussi des gens ordinaires, des femmes en Russie 56 , des bonnes, femmes de ménages et tutti quanti, sans droit aux congés payés ni à l'assurance-chômage 57 , des patients en analyse dont on utilise les récits pour faire un livre, mis lourdement à contribution, sans le moindre espoir d'accès aux droits d'auteur et qui ont cependant déjà payé, fort cher sans doute ${ }^{58}$. Écrire ne doit surtout pas servir à donner bonne conscience, ni à fonder un quelconque pouvoir.

\section{Le risque et le courage de la critique 59}

La critique est un pouvoir, et comme tout pouvoir, elle confere une responsabilité. Depuis son tout premier compte rendu dans Spirale, Suzanne Lamy pose le problème du «Pouvoir du discours et [du] discours de pouvoir»60.

Porte-parole de l'équipe de Spirale lors d'une rencontre organisée par l'Union des écrivains, Suzanne Lamy définit ainsi la mission de la critique: aider le

51 Quand je lis je m'invente, p. 53.

52 Ibid., p. 18.

53 Suzanne Lamy, «De l'utilisation du féminisme», dans Spirale, no 17, mars 1981, p. 3-4; «Un saut (manqué) dans la mêlée», dans Spirale, no 24, avril 1982 , p. 8.

54 Quand je lis je m'invente, p. 90.

55 IBid., p. 94.

56 Suzanne Lamy, «le Sens de l'histoire», dans Spirale, no 16, février 1981, p. 4.

57 Suzanne Lamy, «Thérapie à coups d'épingles», dans Spirale, no 20, juin 1981 , p. 9.

58 «Quand le silence est d'ors, p. 6.

59 Suzanne Lamy, «le Risque et le courage de la critique», présenté à l'Union des écrivains, novembre 1982, p. 1 du manuscrit.

60 Suzanne Lamy, «Pouvoir du discours et discours de pouvoir», dans Spirale, no 6 , janvier 1980 , p. 3 . 
lecteur à lire, à perdre sa naïveté, à voir ce qui est en jeu dans la production culturelle par rapport a la tradition, aux idées reçues, a l'inconscient, d l'idéologie, saisir les enjeux politiques, sociaux, moraux, esthétiques, théoriques 61 . Une telle conception dépasse de loin, on le voit, le travail universitaire traditionnel. Elle situe l'activité critique en plein dans l'espace du politique.

Quelles sont les qualités recherchées, dans le texte critique? Comme pour le texte de création, travail sur l'écriture, audace, érudition. La critique «universitaire» ne l'intéresse guère: J'ai perdu l'habitude de la critique guindée et sûre d'elle-même. Je m'ennuie avec elle: elle ne me dit rien de ce qui m'importe62.

Elle se moque des textes dont la méthode est absente ou trop présente ${ }^{63}$ et des auteurs interchangeables par la bonne conscience donnée par la somme de travail accompli et par la neutralité de ton qui les rend presque uniformes 64 . Inversement, tel autre livre reçoit son approbation parce qu'écrit dans un style enlevé, débridé même, ce qui toutefois ne nuit pas à la qualité de la recherche 65 . Les écrits de René Payant, de Bernard Beugnot, d'André Belleau la séduisent par le ton, la finesse.

Pour éviter le piège de la critique impressionniste, la théorie, le savoir sont indispensables, mais

ce savoir n'est rien s'il n'est accompagné d'un autre savoir, du savoir que nous renvoie l'origine du mot: "sapere», c'est aussi "goûter», «connaître par le goût», par le coeur et par le corps ${ }^{66}$.

Les articles d'un Patrick Imbert amènent Suzanne Lamy à constater que pour un certain formalisme, ce ne sont plus les méthodes qui sont au service des textes, mais les textes au service des grilles. La critique ayant la mission d'amener les lecteurs et lectrices à des textes, d̀ leur connaissance, d̀ leur approfondissement, l'excès de méthode ne pourra que rebuter. Les écrits demeurent des blocs opaques. L'énergie ne circule pas. Aucun déploiement de désir ne porte vers les textes 67.

61 «le Risque et le courage de la critique», p. 1.

62 Quand je lis je m'invente, p. 27.

63 Suzanne Lamy, «Pas assez ou trop de méthode», dans Spirale, no 36, septembre 1983, p. 4.

64 Suzanne Lamy, «Qu'en est-il de l'invention critique?», dans Spirale, no 43, mai 1984 , p. 7.

65 Suzanne Lamy, «Comment se perd la virginité», dans Spirale, no 27, septembre 1982, p. 4.

66 Quand je lis je m'invente, p. 58.

67 «Pas assez ou trop de méthode», p. 4. 
Parmi les dernières publications de Suzanne Lamy, les deux chroniques de revues parues dans Voix \& images expriment de manière saisissante le plaisir qu'elle attend de la critique:

Il est plus facile aujourd'hui qu'hier de dire que ce qui se lit sans plaisir dans l'immédiat risque d'être insupportable d'odeur de ranci dans le futur. Il n'y a aucune obligation décrire et ce qui est paraphrase de paraphrase n'aurait pas dû atteindre le noir de l'imprimé. Ce n'est pas être trop exigeant non plus que de s'attendre d̀ un minimum d'aisance dans la précision et dans l'art de la nuance, de ne pas éprouver la moindre gêne à abandonner tout écrit qui, tout en étant savant, reste englué dans une rebutante lourdeur68.

L'ennui inspire à la lectrice une réaction des plus saines:

Il m'est arrivé de filer à l'anglaise ou bien je me suis simplement endormie [...] Et tant pis si l'engourdissement de l'esprit a gagné celui des paupières. La distinction de l'âme et du corps n'appartient qu'au petit catéchisme. Et l'effet du sommeil irrépressible n'en revient pas forcément au lecteur 69 .

Ce qui l'amène à conclure, avec ironie: Si les bons sentiments ne font pas de la bonne littérature, ils ne font pas non plus de la bonne critique 70 .

\section{La responsabilité de la critique, la responsabilité de l'écrivaine} faire

On l'a vu, le ou la critique littéraire a une responsabilité morale, celle de

entendre clairement à quel niveau de qualité ou d'importance se situe la production recensée, avec tous les risques que cela comporte, d'erreur, de perdre ses amis, de se tailler une réputation de malcommode. Ce courage, il faut l'avoir, je crois 71 .

Elle court le risque de s'attaquer aux plus gros canons, comme la Anne Hébert des Fous de Bassan, parce qu'elle refuse l'aura d'intouchable que confere la consécration d'un auteur au Québec. Dans le cas de Quelle douleur!, de Monique Larouche-Thibault, des commentateurs qui ont la chance de ne pas être effleurés par le doute ont crié au génie 72 , propulsant vers le succès commercial un livre

68 «Au-delà de l'actualité», p. 161.

69 Suzanne Lamy, «De l'érudition : l'esprit et la lettre», dans Voix \& Images, 35 , vol. XII, no 2 , hiver 1987, p. 342.

70 «De l'érudition...», p. 346.

71 «le Risque et le courage de la critique», p. 2.

72 Suzanne Lamy, «les Jeux de massacre, vous aimez?», dans Spirale, no 48, décembre 1984, p. 10. 
au demeurant fort ordinaire; au Québec, on consacre trop vite et sans discernement, Suzanne Lamy ne cesse de le rappeler.

Tenir compte du référent, du pouvoir idéologique, présuppose une responsabilité de l'écrivain-e. Ainsi, le roman suscite la méfiance de Suzanne Lamy en ce qu'il permet d'escamoter l'idéologique:

le je qui écrit s'y sent [...] protégé par les coulisses ou resteront cantonnées les idées, alors qu'à l'avant-scène les personnages dans la diffraction du je (jeu) qui les a produits se pavanent dans une presque totale immunité 73 .

D'immunité, pour Suzanne Lamy, il n'y en a pas, ne peut y en avoir. Elle admire les écrits au féminin, et la critique qui s'y consacre, d'avoir remis le corps à sa place centrale: Corps mis d l'index par le structuralisme. Autant de corps qui sont assurés d'une chose: la parole est acte ${ }^{74}$.

Ainsi, elle passe par le performatif pour en arriver à la responsabilité du critique, mais aussi de l'écrivain-e. En bonne existentialiste, elle croit que chacun de nos actes nous engage, et, à plus forte raison, chacun de nos écrits.

C'est toujours au nom de cette responsabilité qu'elle s'en prend aux écrivains, consacrés ou non, qui lui semblent perpétuer des abus. Le fameux éreintement des Fous de Bassan ne s'intitule-t-il pas précisément «le Roman de l'irresponsabilité»?

Que reproche-t-elle, au juste, à ces Fous de Bassan, reçus comme un pur chef-d'oeuvre par l'ensemble de la critique québécoise? C'est un livre facile, un roman à succès prévisible, à la modernité trompeuse, au style plein de tics et de clichés, et qui, de surcroît, véhicule une idéologie machiste 75 . On le voit, la condamnation est totale. Mais les preuves sont là, des citations, des précisions, avec tout le sérieux voulu.

Adrien de peine et misère, d'Yves Lacroix, est jugé bien-nommé par l'ennui qu'il inspire. Mais le plus grave, c'est qu'il est symptomatique du cas de plusieurs professeurs d'université qui assument difficilement leur culture actuelle, la culture savante, [...] culture à laquelle ils doivent leur sécurité matérielle et la possibilité de s'exprimer 76 . C'est le type d'ambivalence que Suzanne Lamy refuse comme un manque de lucidité.

73 Suzanne Lamy, «Des ambiguités de la fiction à la tentation du récit», présenté au colloque APFUCC, mai 1984, p. 9 du manuscrit.

74 «Au jeu du désir...», p. 82.

75 Suzanne Lamy, «le Roman de l'irresponsabilité», dans Spirale, no 29, ovembre 1982, p. 2-3.

76 Suzanne Lamy, «Adrien le bien-nommé», dans Spirale, no 52, mai 1985, p. 4. 
Mais sa condamnation la plus sévère, elle la réserve à Yolande Villemaire, dont elle avait pourtant porté aux nues la Vie en prose. Pour elle, la Constellation du Cygne est un roman pornographique, anti-féministe; une vision édulcorée de l'Histoire permet d'en évacuer le politique au nom de l'amour. L'indignation de Suzanne Lamy est à son comble lorsqu'elle s'écrie: Qui est Yolande Villemaire pour pardonner ainsi a Hitler 77 ?

À cela, on pourrait répliquer que cette lecture est bien littérale, faire remarquer que le travail de Yolande Villemaire se passe ailleurs, qu'il rejoint un autre plan, qu'il utilise l'histoire comme un simple matériau. À quoi Suzanne Lamy aurait sans doute répondu qu'il n'y a dans le domaine de l'éthique ni audessus, ni au-delà, qu'on ne peut planer en ignorant le réel. La responsabilité de l'écrivain ne peut être éludée, ni celle du ou de la critique qui doit remettre les choses en perspective.

Longue, minutieuse, l'analyse de la Constellation du Cygne frappe par le soin accordé à étayer chaque affirmation. Suzanne Lamy n'était pas de ceux qui aiment le trait facile et gratuit, qui brillent le plus lorsqu'ils ont le moins aimé. Lorsqu'elle démolit un texte, ce n'est jamais de gaieté de coeur. Son style, habituellement aérien, s'appesantit, le bonheur de lecture disparaît. Mais ces choses-là, «il faut que quelqu'un les dise», croyait-elle, même lorsque c'est pénible, risqué.

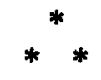

On parle beaucoup de Suzanne Lamy depuis qu'elle est morte. De ses écrits, que restera-t-il? Pour le plaisir de la lecture, ses textes amoureux demeureront. Les textes sévères aussi, pour la justesse du trait, la rigueur de la démonstration, la fermeté de la position politique défendue.

L'humide et le sec, la tête et le corps réunis, voilà de quoi Suzanne Lamy nous a fait cadeau, comme de son écriture alerte, un peu précieuse, toute chaude de sa présence, encore maintenant.

Depuis dix ans seulement, elle avait fait de la critique et de l'écriture son pain quotidien - son premier livre n'est paru qu'en 1977. Dix ans, c'est peu, dans une vie de femme, d'écrivaine, qui commençait à peine à entrevoir tout ce dont elle était capable. Me manquent tous les livres qu'elle n'a pas eu le temps d'écrire, me manque sa voix si juste, si personnelle

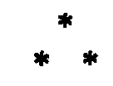

77 Suzanne Lamy, «Du privé au politique : la Constellation du Cygne de Yolande Villemaire», inédit publié dans le présent numéro. 
